\title{
Response to Schell and colleagues
}

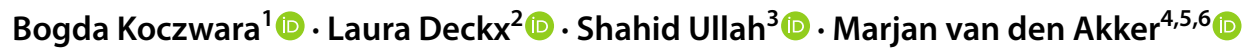

Received: 21 November 2021 / Accepted: 14 December 2021 / Published online: 19 January 2022

(c) The Author(s), under exclusive licence to Springer-Verlag GmbH Germany, part of Springer Nature 2021

Thank you to Schell and colleagues for your interest in our manuscript. We agree that there is a need for further research into the mechanisms of co-existence of cancer and comorbid diseases and the need for development and application of comorbidity assessment and management tools that are validated in the younger cohort. We wish to stress however that our study did not include patients younger than 50 years and thus we are not able to comment on the relationship of cancer and comorbid diseases in individuals younger than 50. Other large cohort studies have demonstrated higher prevalence of comorbidities of survivors of childhood cancer emphasizing the importance of this issue in all cancer survivors and underscoring the importance of research in this area [1]. It is unclear if the etiology and mechanisms for cancer and chronic disease development in paediatric/ adolescent individuals with cancer is similar or different to those in middle age and older adults. Future research including preclinical and animal model research may shed a light into this intriguing area.
Author contribution All authors contributed to the conceptualization and writing.

\section{Declarations}

Consent to publish All authors granted the consent to publish the manuscript.

Conflict of interest Dr Koczwara was a supervisor of Ms Schell who is a medical student at the Flinders University. Dr Koczwara did not solicit Ms Schell's letter nor contributed to it.

\section{Reference}

1. Oeffinger $\mathrm{KC}$ et al (2006) Chronic health conditions in adult survivors of childhood cancer. N Engl J Med 355(15):1572-1582

Publisher's note Springer Nature remains neutral with regard to jurisdictional claims in published maps and institutional affiliations.

Bogda Koczwara

bogda.koczwara@flinders.edu.au

1 Department of Medical Oncology, Flinders Medical Centre and Flinders University, Adelaide, Australia

2 Primary Care Clinical Unit, The University of Queensland, Brisbane, Australia

3 College of Medicine and Public Health, Flinders University, Adelaide, Australia

4 Institute of General Practice, Goethe University, Frankfurt, Germany

5 Academic Center for General Practice, Department of Public Health and Primary Care, KU Leuven, Leuven, Belgium

6 Department of Family Medicine, School CAPHRI, Maastricht University, Maastricht, The Netherlands 